\title{
Positive-definiteness and integral representations for special functions
}

\author{
J. Buescu' ${ }^{1}$ - A. C. Paixão \\ Received: 27 February 2020 / Accepted: 6 August 2020 / Published online: 13 August 2020 \\ (c) Springer Nature Switzerland AG 2020
}

\begin{abstract}
It is known that a holomorphic positive definite function $f$ defined on a horizontal strip of the complex plane may be characterized as the Fourier-Laplace transform of a unique exponentially finite measure on $\mathbb{R}$. In this paper we apply this complex integral representation to specific families of special functions, including the $\Gamma, \zeta$ and Bessel functions, and construct explicitly the corresponding measures, thus providing new insight into the nature of complex positive and co-positive definite functions. In the case of the $\zeta$ function this process leads to a new proof of an integral representation on the critical strip.
\end{abstract}

Keywords Positive definite functions · Fourier-Laplace transform - Characteristic functions · Holomorphy · Gamma function · Zeta function · Exponentially convex functions

Mathematics Subject Classification Primary 42A82; Secondary 30A10 - 30C40 . 60E10

\section{Introduction}

Positive definite functions are a classical subject in Mathematics, having for long attracted attention from different branches of Analysis such as integral equations

The first author acknowledges partial support by Fundação para a Ciência e Tecnologia, UID/MAT/04561/2019.

$凶 \quad$ J. Buescu

jsbuescu@fc.ul.pt

A. C. Paixão

apaixao@dem.isel.ipl.pt

1 Department of Matemática, FCUL and CMAFCIO, Lisbon, Portugal

2 Área Departamental de Matemática, ISEL, Lisbon, Portugal 
(where they generate positive definite kernels), harmonic analysis [3,28] or probability theory [5,26]; for an excellent historical review see [29]. Recent developments have, however, attracted renewed interest in positive definiteness in the complex setting, see e.g. [5,18,20-23,25,31,37]. The classical integral representations of positive definite functions $[15,16]$ have been shown by the authors, in the one dimensional complex case, to induce a bijection between holomorphic positive definite functions and a class of positive measures known as exponentially finite measures [12,13]. This bijection is performed by means of the Fourier-Laplace transform. Moreover it unifies the classical theorems of Bochner on positive definite functions and of Widder on exponentially convex functions: these become special cases of this characterization, corresponding respectively to the real and imaginary sections of the complex integral representation.

The main purpose of this paper is to present, through a collection of explicit examples, the connections of these representations of positive definite functions to properties of regularity and extension of these functions, and to derive consequences of these connections for the characterization of complex variable positive definite functions on horizontal strips of the complex plane. This makes the paper partly expository in nature, since many of the results necessary for context are cited without proof. These results have been published elsewhere by the authors; proofs, unless otherwise stated, may be found in references [6-14]. The results involving special functions, namely the $\Gamma, \zeta$ and Bessel functions, are original.

The paper is structured as follows. The first section will be dedicated to the presentation of basic definitions and relevant properties of the different notions of positive definiteness involved in the subsequent discussion. The conditions under which a complex variable positive definite function defined on a general (horizontal or vertical) strip admits an integral transform representation are next explored. This point of view is then applied to specific cases, including the special functions $\Gamma(z), \zeta(z)$ and Bessel functions of the first kind. As it turns out, $\Gamma$ is alternately co-positive and co-negative definite on neighbouring vertical strips of the complex plane, $\zeta$ is co-positive definite on the half-plane $\mathfrak{R}(z)>1$, and the normalized Bessel functions are co-positive definite on the entire complex plane. For these functions we compute explicitly the exponentially finite measures relative to which the integral representation holds, and as a corollary we derive a new proof of a known integral representation of the $\zeta$ function on the critical strip.

\section{Real variable positive definite functions}

A brief review of the properties of real variable positive definite functions relevant for our purposes will be performed below.

A function $f: \mathbb{R} \rightarrow \mathbb{C}$ is positive definite if

$$
\sum_{k, l=1}^{n} f\left(x_{k}-x_{l}\right) \xi_{k} \bar{\xi}_{l} \geq 0
$$


for every choice of $x_{1}, \ldots x_{n} \in \mathbb{R}$ and $\xi_{1}, \ldots, \xi_{n} \in \mathbb{C}$; that is, if every matrix $\left[f\left(x_{k}-\right.\right.$ $\left.\left.x_{l}\right)\right]_{k, l=1}^{n}$ is positive semidefinite.

Basic properties of positive definite functions include the following well-known facts:

1. $f(0) \geq 0$ and $f(-x)=\overline{f(x)} \forall x \in \mathbb{R}$,

2. $|f(x)| \leq f(0) \forall x \in \mathbb{R}$.

These follow immediately from (2.1) by consideration of order 1 and 2 matrices; references to these properties may be found in $[3,30]$. We stress that these are purely algebraic properties that hold under the single hypothesis of positive definiteness and for which no form of regularity is necessary. Under the further hypothesis of continuity, positive definite functions satisfy the following classical representation theorem.

Theorem 2.1 (Bochner) A continuous function $f: \mathbb{R} \rightarrow \mathbb{C}$ is positive definite if and only if it is the Fourier transform of a unique, finite, non-negative measure $\mu$ on $\mathbb{R}$, that is

$$
f(x)=\int_{-\infty}^{+\infty} e^{i t x} d \mu(t)
$$

The Bochner representation (2.2) is often the most expedite way to prove that a function is positive definite. Indeed, the most obvious class of positive definite functions are characteristic functions, which arise naturally in probability theory as Fourier transforms of probability measures. Specific examples follow immediately: functions like $\cos (a x), e^{-|x|}, \frac{1}{1+x^{2}}, e^{-x^{2}}$ are Fourier transforms of probability measures; consequently, from the Bochner representation theorem, they are positive definite.

A first case of propagation of regularity concerns the consequences of certain regularity assumptions for a real variable positive definite function on a neighborhood of the origin.

Theorem 2.2 Let $\phi$ be a positive definite function on $\mathbb{R}$. Suppose $\phi$ is of class $C^{2 k}$ on a neighborhood of the origin. Then $\phi$ is $C^{2 k}(\mathbb{R})$.

This is a classical result for which several proofs exist. The more usual proof relies in standard properties of the Fourier transform and may be found, for instance, in [17]. A constructive approach based on algebro-differential properties arising directly from the definition have been given by the authors in [10] or [14].

\section{Holomorphic extensions}

We next turn to an extension result which states that it is possible to extend a realanalytic positive definite function as a holomorphic function to a maximal horizontal strip of the complex plane. This is somewhat of a 'folklore' theorem and priority seems difficult to establish; for proofs see [5,26,30,31]. A different proof, along constructive lines, is given by the authors in [12]. 
Definition 3.1 A horizontal strip on the complex plane is an open set of the form

$$
S_{a, b}=\{z \in \mathbb{C}: a<\Im(z)<b\} \text { for } a, b \in \overline{\mathbb{R}} .
$$

Analogously, a vertical strip on the complex plane is a set of the form

$$
T_{a, b}=\{z \in \mathbb{C}: a<\mathfrak{R}(z)<b\} \text { for } a, b \in \overline{\mathbb{R}} .
$$

Theorem 3.2 Suppose $f: \mathbb{R} \rightarrow \mathbb{C}$ is a positive definite function which is analytic in a neighborhood of the origin. Then $f$ is analytic in $\mathbb{R}$ and there exist $\alpha, \beta \in(0,+\infty]$ such that $f$ extends to a function which is holomorphic in the horizontal strip $S_{-\alpha, \beta}$, with $\alpha$ and $\beta$ maximal with respect to this property. If $\alpha<\infty$ (resp. $\beta<\infty$ ), then $-i \alpha$ (resp. $i \beta)$ is a singularity of this extension, which may be defined on this strip by

$$
\tilde{f}(z)=\int_{-\infty}^{\infty} e^{i z t} d \mu(t), \quad-\alpha<\Im(z)<\beta,
$$

where $\mu$ is a finite non-negative measure on $\mathbb{R}$.

Further reference to related results and generalizations may be found in [13].

With a view to addressing another line of holomorphic extension results with special significance to the contents of the next section, we now turn our attention to real variable co-positive definite functions or, as they are traditionally referred to, exponentially convex functions.

Definition 3.3 A function $G: I \rightarrow \mathbb{C}$, with $I \subset \mathbb{R}$, is said to be co-positive definite if, for every $n \in \mathbb{N}$ and every finite collection $\left\{x_{j}\right\}_{j=1}^{n}$ such that $x_{k}+x_{l} \in I$ for all $k, l,=1, \ldots, n$ we have

$$
\sum_{k, l=1}^{n} G\left(x_{k}+x_{l}\right) \xi_{k} \bar{\xi}_{l} \geq 0
$$

for every finite collection $\left\{\xi_{j}\right\}_{j=1}^{n} \subset \mathbb{C}$.

Co-positive definite functions are most usually defined in the case where $I$ is an interval, in which case they are known in the literature as exponentially convex functions. In the real-variable context, these functions have been introduced by Bernstein in 1929 [4] and are closely related to absolutely monotone functions; they have since been studied by Widder [36], Devinatz [15,16], among other authors.

A number of properties may be established for co-positive definite functions as a direct algebraic consequence of definition 3.3. Notice, for instance, that consideration of the case $n=1$ immediately implies that $G$ is non-negative on $I$. Much more, however, can be said if we assume continuity of $G$ in an open interval. Widder's theorem $([24,36])$, which we rephrase in a more convenient form, asserts that the co-positive definite function $G$ admits the following integral representation. 
Theorem 3.4 (Widder) Let $I=(a, b)$. A function $G: I \rightarrow \mathbb{R}$ may be represented in the form

$$
G(y)=\int_{-\infty}^{+\infty} e^{-y t} d \mu_{I}(t), \quad y \in(a, b)
$$

where $\mu_{I}$ is a non-negative Lebesgue-Stieltjes measure, if and only if $G$ is continuous and co-positive definite on $I$.

From Theorem 3.4 we may deduce the following

Corollary 3.5 Suppose $G(x)$ is a continuous co-positive definite function on the interval $I=(a, b)$. Then $G$ has a holomorphic extension $g(z)$ to the vertical strip $T_{a, b}$ defined by

$$
g(z)=\int_{-\infty}^{+\infty} e^{-z t} d \mu_{I}(t)
$$

Furthermore, we have

$$
\frac{d^{n}}{d z^{n}} g(z)=\int_{-\infty}^{+\infty}(-t)^{n} e^{-z t} d \mu_{I} \forall n \in \mathbb{N}
$$

The contents and interplay of Theorems 3.2 and 3.4 may be viewed as the natural motivation for the definition of positive and co-positive definite functions of a complex variable. This is the main concern of the next section, along with the study of corresponding consequences for the integral representation of these functions.

\section{Complex variable positive and co-positive definite functions}

We begin with a short introduction to complex variable positive definite functions. A more comprehensive discussion may be found in [11,12].

Definition 4.1 A set $S \subset \mathbb{C}$ (resp. $T \subset \mathbb{C}$ ) is called a codifference set (resp. cosum set) if there exists a set $\Omega \subset \mathbb{C}$ such that $S$ may be written as

$$
S=\Omega-\bar{\Omega} \equiv\left\{z \in \mathbb{C}: \exists z_{1}, z_{2} \in \Omega: z=z_{1}-\overline{z_{2}}\right\}
$$

resp.

$$
T=\Omega+\bar{\Omega} \equiv\left\{z \in \mathbb{C}: \exists z_{1}, z_{2} \in \Omega: z=z_{1}+\overline{z_{2}}\right\}
$$

We write $S=\operatorname{codiff}(\Omega)(\operatorname{resp} . T=\operatorname{cosum}(\Omega))$.

Basic but very useful examples of codifference and cosum sets are, respectively, horizontal and vertical strips $S_{a, b}$ and $T_{a, b}$ as in Definition 3.1. 
The central notions of a positive or co-positive definite function of a complex variable are the concern of the next definition. A somewhat more general version of these concepts, bearing no additional significance in the context of this paper, may be found in [12].

Definition 4.2 Let $\Lambda \subset \mathbb{C}$ be a codifference (resp. cosum) set. A function $f: \Lambda \rightarrow \mathbb{C}$ is said to be positive definite (resp. co-positive definite)in $\Lambda$ if, for every $n \in \mathbb{N}$ and every finite collection $\left\{z_{j}\right\}_{j=1}^{n}$ such that $z_{k}-\overline{z_{l}} \in S$ for all $k, l=1, \ldots, n$ (resp. $z_{k}+\overline{z_{l}} \in \Lambda$ for all $\left.k, l=1, \ldots, n\right)$, we have

$$
\sum_{k, l=1}^{n} f\left(z_{k}-\overline{z_{l}}\right) \xi_{k} \overline{\xi_{l}} \geq 0
$$

resp.

$$
\sum_{k, l=1}^{n} f\left(z_{k}+\overline{z_{l}}\right) \xi_{k} \overline{\xi_{l}} \geq 0
$$

for every collection $\left\{\xi_{j}\right\}_{j=1}^{n} \subset \mathbb{C}$, that is, if every matrix $\left[f\left(z_{k}-\overline{z_{l}}\right)\right]_{k, l=1}^{n}$ (resp. $\left.\left[f\left(z_{k}+\overline{z_{l}}\right)\right]_{k, l=1}^{n}\right)$ is positive semidefinite.

Remark 4.3 We observe that, for a positive definite function $f$ on a horizontal strip $S_{a, b}$, the real variable function $F_{y}(x)=f(x+i y)$ for fixed $y \in(a, b)$ verifies the condition $\sum_{k, l=1}^{n} F_{y}\left(x_{k}-x_{l}\right) \xi_{k} \bar{\xi}_{l} \geq 0$ for every collection $\left\{x_{j}\right\}_{j=1}^{n} \subset \mathbb{R}$, every collection $\left\{\xi_{j}\right\}_{j=1}^{n} \subset \mathbb{C}$ and every positive integer $n$, and is thus a positive definite real-variable function. On the other hand, the function $G(y)=f(i y)$, for $y \in(a, b)$, satisfies the inequalities $\sum_{k, l=1}^{n} G\left(x_{k}+x_{l}\right) \xi_{k} \bar{\xi}_{l} \geq 0$. We recognize this property as the real-variable co-positive definiteness condition.

Remark 4.4 It is now easily seen, from these definitions, that the holomorphic function defined by (3.3) is co-positive definite on $T_{a, b}$.

These definitions of cosum set and co-positive definite function are readily seen to be closely related to those of codifference set and positive definite function, respectively. More precisely, the following is true.

Proposition 4.5 We have:

(i) $T \subset \mathbb{C}$ is a cosum set if and only if $S=i T$ is a codifference set.

(ii) $f(z)$ is positive definite in a set $S \subset \mathbb{C}$ if and only if $g(z)=f(i z)$ is co-positive definite in $T=-i S$.

It is immediate to show that $S_{a, b}$ is a horizontal strip if and only $i S_{a, b}$ is a vertical strip. Note also that Definition 4.2 does not require any regularity on the function $f$. Even in this context, a number of properties of positive definite functions may be established as purely algebraic consequences of Definition 4.2, as the next proposition shows for positive definite functions. 
Proposition 4.6 Let $f$ be a positive definite function on a codifference set $S \subset \mathbb{C}$. Then the following hold.

(1) If $x, y \in \mathbb{R}$ and $\pm x+i y \in S$, then $f(-x+i y)=\overline{f(x+i y)}$. In particular, in the special case where $x=0$ (implying that iy $\in S$ ) it follows that $f(i y)$ is real and non-negative.

(2) If $x, y, \beta \in \mathbb{R}$ and $\pm x+i y, i(y \pm \beta) \in S$, then

$$
|f(x+i y)|^{2} \leq f(i(y-\beta)) f(i(y+\beta)) .
$$

(3) If $f$ is positive definite, then so is $\bar{f}$.

(4) If $f_{1}, \ldots, f_{n}$ are positive definite functions and $c_{i} \geq 0$, then so is $\sum_{k=1}^{n} c_{k} f_{k}$.

(5) If $\left\{f_{n}\right\}_{n \in \mathbb{N}}$ is a pointwise convergent sequence of positive definite functions, then the limit $f(z)=\lim _{n \rightarrow \infty} f_{n}(z)$ is a positive definite function.

(6) The product of positive definite functions is positive definite.

Proofs of these properties may be found in [12], Proposition 4.6, and [13], Proposition 1.3. Property (2) is sometimes called the ridge property for complex positive definite functions $[26,38]$. Corresponding properties for co-positive definite functions immediately follow from proposition 4.5.

We close this section with a reference to non-trivial examples of functions defined on strips of the complex plane satisfying some kind of positive or negative definiteness condition. In order to avoid ambiguity with respect to other areas of the literature, e.g. Harmonic Analysis (see e.g. [3]), it is important to specify the precise sense in which we use the term 'negative definite function'.

Definition 4.7 Let $\Lambda \subset \mathbb{C}$. A function $f: \Lambda \rightarrow \mathbb{C}$ is said to be

(i) negative definite in $\Lambda$ if $g=-f$ is positive definite in $\Lambda$;

(ii) co-negative definite in $\Lambda$ if $g=-f$ is co-positive definite in $\Lambda$.

The following examples illustrate the richness of behaviour of complex-variable positive definite functions.

Example 1 The function $f(z)=\frac{1}{1+z^{2}}$ is positive definite in the horizontal strip $S_{-1,1}$ and negative definite in the half-planes $S_{-\infty,-1}$ and $S_{1, \infty}$. Let $j$ and $n$ be positive integers and define $a_{j}=j$ for $j \leq n, a_{n+1}=\infty$. Since the product of positive definite functions is positive definite, it follows that

$$
f_{n}(z)=\prod_{j=1}^{n} \frac{1}{\left(1-i \frac{z}{a_{j}}\right)\left(1+i \frac{z}{a_{j}}\right)}
$$

is positive definite in the central horizontal strip $S_{-1,1}$, alternating between negative and positive definite in neighboring horizontal strips $S_{a_{j}, a_{j+1}}$ and $S_{-a_{j+1},-a_{j}}$ for $j=$ $1, \ldots, n$ (Fig. 1). 


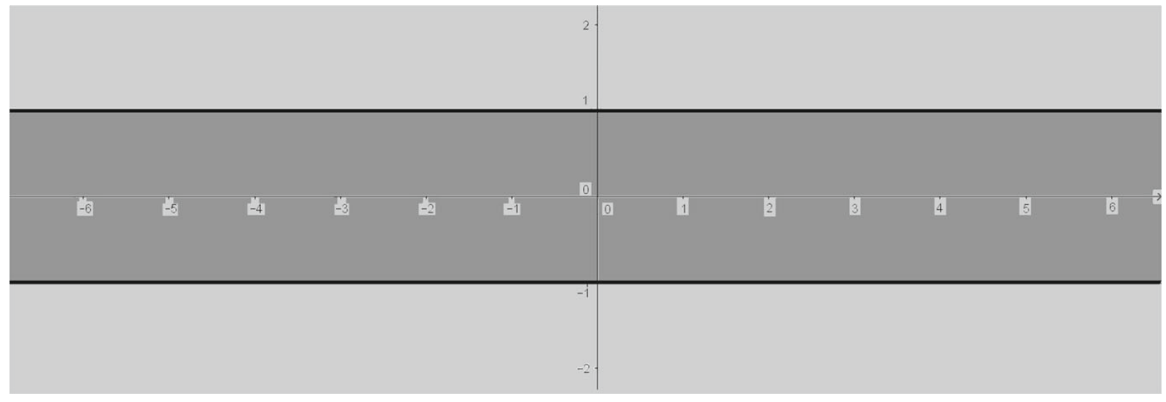

Fig. $1 f(z)=\frac{1}{1+z^{2}}$ is positive definite in the horizontal strip $S_{-1,1}$ and negative definite in the complementary half-planes

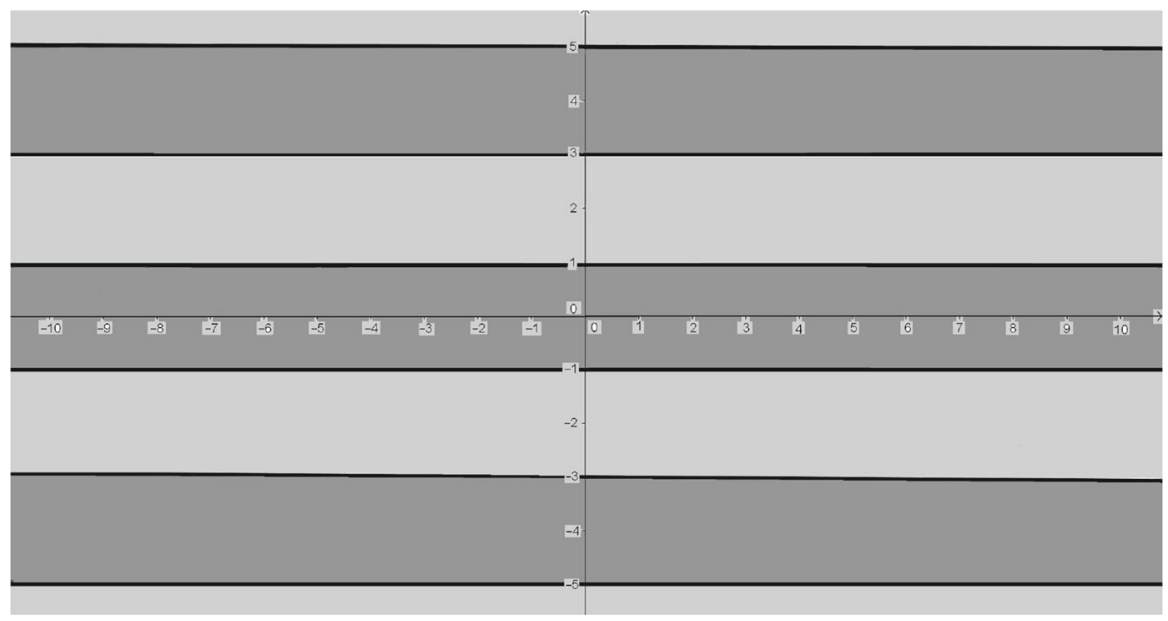

Fig. $2 f(z)=\frac{1}{\cosh \left(\frac{\pi z}{2}\right)}$ is positive definite in the dark horizontal strips and negative definite on the light horizontal strips

Example 2 The function $f(z)=\frac{1}{\cosh \left(\frac{\pi z}{2}\right)}$ is positive definite in any horizontal strip of the form $S_{4 n-1,4 n+1}$ and negative definite in any horizontal strip $S_{4 n+1,4 n+3}$ for $n \in \mathbb{Z}$. In fact, the well-known integral representation (see e.g. [23])

$$
f(z)=\frac{1}{\cosh \left(\frac{\pi z}{2}\right)}=\frac{1}{\pi} \int_{-\infty}^{\infty} \frac{1}{\cosh t} e^{i z t} d t
$$

implies that it is positive definite on the strip $S_{-1,1}$. It is not difficult to show directly that $f$ is negative definite on $S_{1,3}$. Since $f$ is periodic of period $4 i$, it alternates between being positive definite on horizontal strips $S_{4 n-1,4 n+1}$ and negative definite on horizontal strips $S_{4 n+1,4 n+3}$ (Fig. 2). 


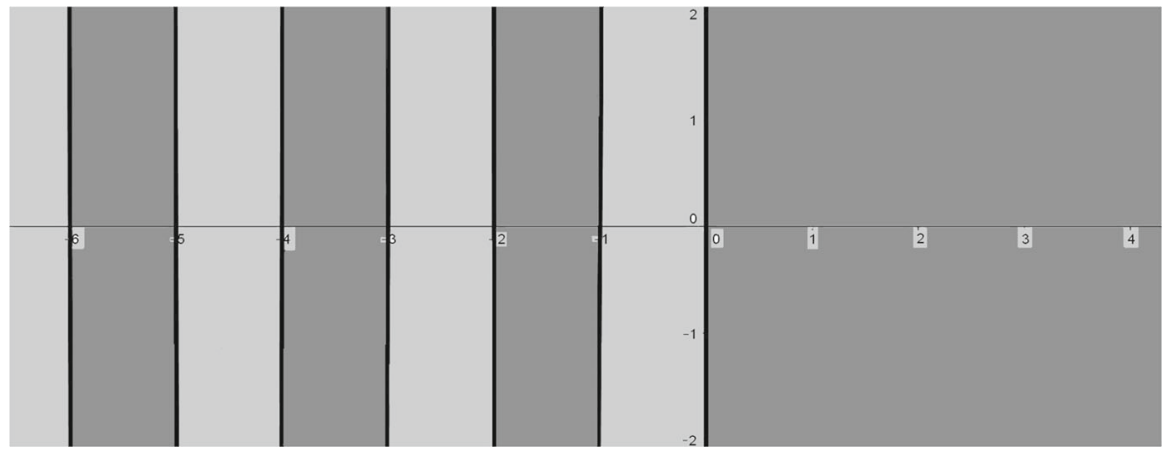

Fig. 3 The $\Gamma$ function is co-positive definite on the half-plane $\Re(z)>0$ and in the dark shaded vertical strips, and co-negative definite on the light vertical strips

Example 3 The $\Gamma$ function, defined by

$$
\Gamma(z)=\int_{0}^{+\infty} x^{z-1} e^{-x} d x
$$

for $\Re(z)>0$, is easily seen to satisfy Definition 4.2 , so it is co-positive definite in that half-plane. The $\Gamma$ function has a simple pole at $z=0$ and at every negative integer. In each vertical strip $T_{-n-1,-n}$, for integer $n \geq 0$, the $\Gamma$ function satisfies the Cauchy-Saalschütz formula (see e.g. [35], pg. 244)

$$
\Gamma(z)=\int_{0}^{+\infty} x^{z-1}\left(e^{-x}-\sum_{m=0}^{n}(-1)^{m} \frac{x^{m}}{m !}\right) d x
$$

from which a simple calculation shows that it is co-negative definite on the vertical strip $T_{-1,0}$, alternating from co-negative definite to co-positive definite and back in neighbouring vertical strips. Corresponding statements are of course valid for positivedefiniteness of $\Gamma(-i z)$ on horizontal strips in view of Proposition 4.5.

It is easily shown that the part of the integrand function in parentheses switches sign with each strip transition: it is positive on the strips where $\Gamma$ is co-positive definite and negative on the strips where $\Gamma$ is co-negative definite. That this necessarily happens is of course an immediate consequence of Bochner's theorem through a global change of sign (Fig. 3).

Example 4 Consider the real-variable Bessel function of the first kind $J_{\alpha}(x)$. For $\alpha>$ $-\frac{1}{2}$ the normalized Bessel function $\mathcal{J}_{\alpha}(x)$ is defined by

$$
\mathcal{J}_{\alpha}(x)=2^{\alpha} \Gamma(\alpha+1) x^{-\alpha} J_{\alpha}(x)
$$


and extends to a real-analytic in $\mathbb{R}$ with $\mathcal{J}_{\alpha}(0)=1$, see $[1,2,27,32]$. This function satisfies the integral representation

$$
\mathcal{J}_{\alpha}(x)=\frac{1}{2^{\alpha-1} \sqrt{\pi} \Gamma(\alpha+1 / 2)} \int_{0}^{1}\left(1-t^{2}\right)^{\alpha-1 / 2} \cos (x t) d t,
$$

see e.g. $[19,34]$. This representation admits the natural extension to the complex plane

$$
\mathcal{J}_{\alpha}(z)=\frac{1}{2^{\alpha-1} \sqrt{\pi} \Gamma(\alpha+1 / 2)} \int_{0}^{1}\left(1-t^{2}\right)^{\alpha-1 / 2} \cos (z t) d t .
$$

This integral may be rewritten in the form of a Fourier-Laplace transform (see Example 10), and application of Theorem 5.5 below shows that $\mathcal{J}_{\alpha}$ is an entire complex-variable positive definite function. This implies, for integer $\alpha \geq 0$, that

$$
J_{\alpha}(z)=\frac{1}{2^{\alpha} \Gamma(\alpha+1)} z^{\alpha} \mathcal{J}_{\alpha}(z)
$$

defines an entire extension $J_{\alpha}$ to the complex plane. If $\alpha=0$, in particular, we have $J_{0}(z)=\mathcal{J}_{0}(z)$, which provides a novel proof of the fact that $J_{0}$ is an entire positive definite function.

Example 5 Riemann's zeta function $\zeta(z)$ is defined by $\zeta(z)=\sum_{n=1}^{\infty} \frac{1}{n^{z}}$ for $z \in T_{1,+\infty}$. Each summand in the series $\frac{1}{n^{z}}=e^{-z \ln n}$ is co-positive definite and therefore $\zeta(z)=$ $\sum_{n=1}^{\infty} \frac{1}{n^{z}}$ is co-positive definite on the half-plane of convergence $T_{1,+\infty}$ by Proposition 4.6. The $\zeta$ function is meromorphic and has a unique simple pole at $z=1$. However, in contrast to previous examples, $\zeta(z)$ is neither co-positive definite nor co-negative definite in any open cosum set in the half-plane $T_{-\infty, 1}=\{z \in \mathbb{C}: \Re(z)<1\}$ since otherwise, for real $x, \zeta(x)$ would be co-positive (resp. co-negative) definite on some open interval $I \subset(-\infty, 1)$ (refer to [12] and use Proposition 4.5 to derive the relevant properties of cosum sets). By maximality of the corresponding holomorphy strip (see e.g. [13], Theorem 2.13), $\zeta(z)$ would necessarily be co-positive (resp. co-negative) in the whole half-plane $T_{-\infty, 1}$. The existence of the trivial zeros of the zeta function would then imply, by the ridge property (statement (2) in Proposition 4.6), that $\zeta(z)$ would vanish identically in the whole half-plane $T_{-\infty, 1}$, which is not the case (Fig. 4).

Example 6 Define the function

$$
\mathcal{Z}(z)=-\frac{\zeta(z)}{z}
$$

It is not difficult to show that $\mathcal{Z}$ is co-negative definite on the half-plane $\Re(z)>1$, co-positive definite on the vertical strip $0<\Re(z)<1$ and neither co-positive neither co-negative on the half-plane $\Re(z)<0$ (Fig. 5).

A full discussion of these properties of $\mathcal{Z}$ is deferred to Example 13, where the interest of introducing this function will become apparent. 


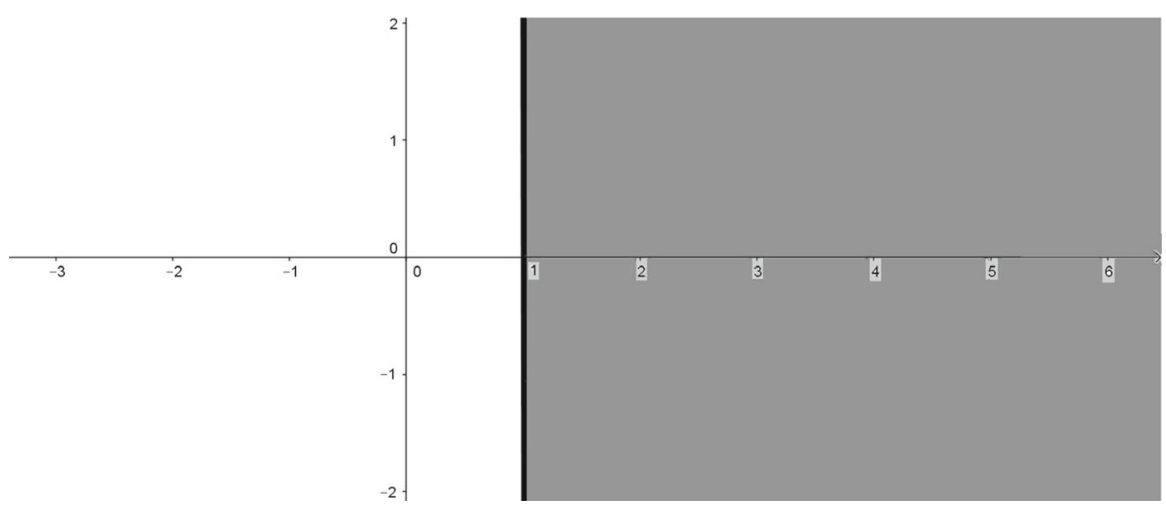

Fig. 4 The $\zeta$ function is co-positive definite on the half-plane $\Re(z)>1$. On the complementary half-plane it is neither co-positive nor co-negative definite

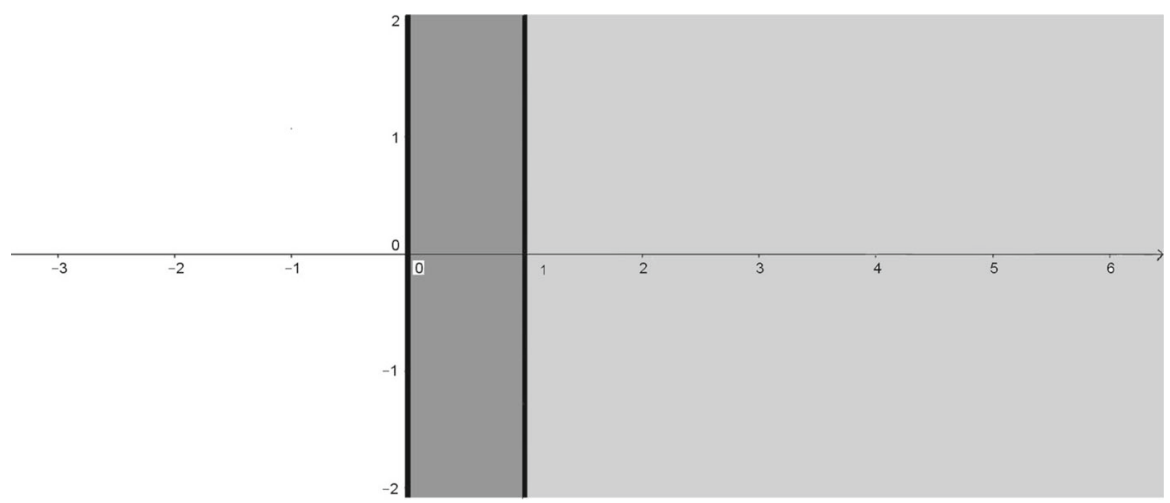

Fig. 5 The $\mathcal{Z}$ function is co-negative definite on the half-plane $\Re(z)>1$ (light grey) and co-positive definite on the critical strip $0<\Re(z)<1$ (dark grey). On the half-plane $\Re(z)<0$ (white) it is neither co-positive nor co-negative definite

\section{Positive definite functions as Fourier-Laplace transforms}

The concept of exponentially finite measure, which we now introduce, will prove essential for what follows.

Definition 5.1 A non-negative $\mu$ is said to be exponentially finite with respect to the (non-empty) interval I if $\int_{-\infty}^{+\infty} e^{-y t} d \mu(t)$ is finite for every $y \in I$.

Remark 5.2 It is easily seen that an exponentially finite measure is a Lebesgue-Stieltjes measure.

Under the hypothesis of holomorphy, the notions of positive and co-positive definiteness reviewed in the previous sections may now be related in the following way.

Theorem 5.3 Suppose $f$ is a holomorphic complex function defined on the horizontal strip $S_{a, b}$. Define $F_{y}(x)=f(x+i y)$ for fixed $y \in(a, b)$ and $x \in \mathbb{R}$, and $G(y)=f(i y)$ for $y \in(a, b)$. Then the following statements are equivalent: 
(1) $f$ is a complex-variable positive definite function on $S_{a, b}$.

(2) There exists a non-negative Lebesgue-Stieltjes measure $\mu$ on $\mathbb{R}$ such that

$$
f(z)=\int_{-\infty}^{+\infty} e^{i t z} d \mu(t) \quad \forall z \in S_{a, b} .
$$

(3) $F_{y}(x)$ is a positive definite function of the real variable $x$ for some $y \in(a, b)$.

(4) There exists a non-negative finite measure $\mu_{y}$ on $\mathbb{R}$ such that

$$
F_{y}(x)=\int_{-\infty}^{+\infty} e^{i t x} d \mu_{y}(t) \quad \forall x \in \mathbb{R}
$$

for some $y \in(a, b)$.

(5) $G(y)$ is a co-positive definite function of the real variable y on the interval $(a, b)$.

(6) There exists a non-negative Lebesgue-Stieltjes measure $\mu_{I}$ on $\mathbb{R}$ such that

$$
G(y)=\int_{-\infty}^{+\infty} e^{-y t} d \mu_{I}(t) \quad \forall y \in(a, b) .
$$

Furthermore, in any of these conditions we also have:

(i) The measures $\mu=\mu_{I}$ and $\mu_{y}$ are uniquely determined by the function $f$.

(ii) $\mu=\mu_{I}$ is exponentially finite with respect to $I=(a, b)$ and $\mu_{y}$ is exponentially finite with respect to $(a-y, b-y)$.

(iii) All the functions $f(z), F_{y}(x)$ and $G(y)$ are infinitely differentiable and may be differentiated under the integral sign in their respective representations (2), (4) and (6).

Theorem 5.3, whose proof may be found in [13], may be seen as a consequence of the interplay of Theorems 3.2 and 3.4 (along with Corollary 3.5) which reveals, through consideration of the complex variable setting, the precise joint significance of these individual results.

We are now ready to present a characterization of holomorphic complex variable positive definite functions on a horizontal strip. The next definition will be useful for that purpose.

Definition 5.4 Let $\mu$ be a non-negative measure on $\mathbb{R}$. We define the complex variable function

$$
f(z)=\int_{-\infty}^{+\infty} e^{i z t} d \mu(t)
$$

to be the Fourier-Laplace transform of $\mu$, and denote it by $f=\mathcal{F} \mathcal{L}(\mu)$.

Theorem 5.5 A function $f$ defined on a horizontal strip $S_{a, b}$ is a holomorphic positive definite function if and only if it is the Fourier-Laplace transform of an exponentially finite measure with respect to the interval $I=(a, b)$. Furthermore, the measure $\mu$ is uniquely determined by $f$.

The proof of this result may be also found in [13]. 


\subsection{Examples}

In order to show how Theorem 5.5 applies to positive and negative definite functions, we now revisit some of the examples in Sect. 4.

Example 7 Consider the case $n=1$ in example (1), i.e., the holomorphic function

$$
f(z)=\frac{1}{1+z^{2}}=\frac{1}{(1+i z)(1-i z)}=\frac{1}{2}\left(\frac{1}{1-i z}+\frac{1}{1+i z}\right) .
$$

For $c \in \mathbb{R}$, define $f_{c}(z)=\frac{1}{c+i z}$ for $z \neq i c$. Define also

$$
\sigma_{c}^{r}(t)=\left\{\begin{array}{ll}
0 & \text { if } t<0 \\
e^{c t} & \text { if } t \geq 0
\end{array}, \quad \sigma_{c}^{l}(t)=\left\{\begin{array}{ll}
e^{c t} & \text { if } t<0 \\
0 & \text { if } t \geq 0
\end{array},\right.\right.
$$

and consider the non-negative Lebesgue-Stieltjes measures defined by

$$
\left.\left.\left.\left.\mu_{c}^{r}(] a, b\right]\right)=\int_{a}^{b} \sigma_{c}^{r}(t) d t, \quad \mu_{c}^{l}(] a, b\right]\right)=\int_{a}^{b} \sigma_{c}^{l}(t) d t .
$$

It is easily shown that $\mu_{c}^{r}$ (resp. $\mu_{c}^{l}$ ) is exponentially finite with respect to ]c, $+\infty$ [ (resp. ] $-\infty, c[$ ). The Fourier-Laplace transforms of these measures may be used to represent $f_{c}(z)$ in the following way:

$$
f_{c}(z)=\frac{1}{c+i z}=\left\{\begin{array}{l}
-\int_{-\infty}^{+\infty} e^{i z t} d \mu_{c}^{r}(t)=-\mathcal{F} \mathcal{L}\left(\mu_{c}^{r}\right) \quad \text { if } \Im(z)>c \\
\int_{-\infty}^{+\infty} e^{i z t} d \mu_{c}^{l}(t)=\mathcal{F} \mathcal{L}\left(\mu_{c}^{l}\right) \quad \text { if } \Im(z)<c
\end{array}\right.
$$

Hence $f_{c}(z)$ is negative definite on $S_{c,+\infty}$ and positive definite on $S_{-\infty, c}$. Observe that $-f_{c}(z)$ has the exact opposite behaviour on these half-planes.

Now, according to identity (5.1), we may write $f(z)=\frac{1}{2}\left(f_{1}(z)-f_{-1}(z)\right)$. By choosing the appropriate equalities and values of $c$ in (5.2), we obtain the following representation for $f$.

(i) if $z \in S_{1,+\infty}$,

$$
f(z)=\frac{1}{2}\left(-\mathcal{F} \mathcal{L}\left(\mu_{1}^{r}\right)+\mathcal{F} \mathcal{L}\left(\mu_{-1}^{r}\right)\right)=\frac{1}{2} \mathcal{F} \mathcal{L}\left(-\mu_{1}^{r}+\mu_{-1}^{r}\right) .
$$

Writing $\mu_{1,+\infty}=\frac{1}{2}\left(-\mu_{1}^{r}+\mu_{-1}^{r}\right)$ and $\sigma_{1,+\infty}=\frac{1}{2}\left(-\sigma_{1}^{r}+\sigma_{-1}^{r}\right)$, we have that $f(z)=\mathcal{F} \mathcal{L}\left(\mu_{1,+\infty}\right)$, where

$$
\sigma_{1,+\infty}= \begin{cases}0 & \text { if } t<0 \\ -\sinh (t) & \text { if } t \geq 0\end{cases}
$$

is the density function associated with $\mu_{1,+\infty}$, and we conclude that $f$ is negative definite in $S_{1,+\infty}$. 
(ii) if $z \in S_{-1,1}$,

$$
f(z)=\frac{1}{2}\left(\mathcal{F} \mathcal{L}\left(\mu_{1}^{l}\right)+\mathcal{F} \mathcal{L}\left(\mu_{-1}^{r}\right)\right)=\frac{1}{2} \mathcal{F} \mathcal{L}\left(\mu_{1}^{l}+\mu_{-1}^{r}\right) .
$$

Writing $\mu_{-1,1}=\frac{1}{2}\left(\mu_{1}^{l}+\mu_{-1}^{r}\right)$ and $\sigma_{-1,1}=\frac{1}{2}\left(\sigma_{1}^{l}+\sigma_{-1}^{r}\right)$, we have $f(z)=$ $\mathcal{F} \mathcal{L}\left(\mu_{-1,1}\right)$, where

$$
\sigma_{-1,1}=\left\{\begin{array}{ll}
\frac{1}{2} e^{t} & \text { if } t<0 \\
\frac{1}{2} e^{-t} & \text { if } t \geq 0
\end{array}=\frac{1}{2} e^{-|t|}\right.
$$

is the density function associated with $\mu_{-1,1}$, and we conclude that $f$ is positive definite in $S_{-1,1}$.

(iii) if $z \in S_{-\infty,-1}$,

$$
f(z)=\frac{1}{2}\left(\mathcal{F} \mathcal{L}\left(\mu_{1}^{l}\right)-\mathcal{F} \mathcal{L}\left(\mu_{-1}^{l}\right)\right)=\frac{1}{2} \mathcal{F} \mathcal{L}\left(\mu_{1}^{l}-\mu_{-1}^{l}\right) .
$$

Writing $\mu_{-\infty,-1}=\frac{1}{2}\left(\mu_{1}^{l}-\mu_{-1}^{l}\right)$ and $\sigma_{-\infty,-1}=\frac{1}{2}\left(\sigma_{1}^{l}-\sigma_{-1}^{l}\right)$, we now have $f(z)=\mathcal{F} \mathcal{L}\left(\mu_{-\infty,-1}\right)$, where

$$
\sigma_{-\infty,-1}= \begin{cases}\sinh (t) & \text { if } t<0 \\ 0 & \text { if } t \geq 0\end{cases}
$$

is the density function associated with $\mu_{-\infty,-1}$, and we conclude that $f$ is negative definite in $S_{-\infty,-1}$.

Notice that the measures representing $f$ on each of these sets are exponentially finite with respect to the intervals $] 1,+\infty[]-1,,1[$ and $]-\infty,-1[$, respectively.

Example 8 Consider the function $f(z)=\frac{1}{\cosh \left(\frac{\pi z}{2}\right)}$ as defined in example 2. From the identity (4.1) we may conclude that $f(z)=\mathcal{F} \mathcal{L}\left(\mu_{0}\right)$ in $S_{-1,1}$, where $\mu_{0}$ is the exponentially finite measure with respect to ] $-1,1[$ associated with the density function $\sigma_{0}(t)=\frac{1}{\pi \cosh t}$. Observing that, for each $n \in \mathbb{Z}, f(z+(2+4 n) i)=-f(z)$ and $f(z+4 n i)=f(z)$, by using the appropriate change of variable we may conclude from (4.1) that:

(i) $f(z)=\mathcal{F} \mathcal{L}\left(\mu_{2+4 n}\right)$ for $z \in S_{1+4 n, 3+4 n}$ associated with the density function $\sigma_{2+4 n}(t)=-\frac{e^{(2+4 n) t}}{\pi \cosh t}, n \in \mathbb{Z}$

(ii) $f(z)=\mathcal{F} \mathcal{L}\left(\mu_{4 n}\right)$ for $z \in S_{-1+4 n, 1+4 n}$, where $\mu_{4 n}$ is the non-negative exponentially finite measure with respect to the interval ] $-1+4 n, 1+4 n$ [ associated with the density function $\sigma_{4 n}(t)=\frac{e^{4 n t}}{\pi \cosh t}, n \in \mathbb{Z}$.

Example 9 Consider the $\Gamma$ function, as defined in Example 3. Notice that, for $\Re(z)>0$, it may be written in the form

$$
\Gamma(z)=\int_{0}^{\infty} e^{z \ln x} \frac{e^{-x}}{x} d x
$$


Performing the change of variable $t=-\ln x$ we may write

$$
\Gamma(z)=\int_{+\infty}^{-\infty} e^{-z t} \frac{e^{-e^{-t}}}{e^{-t}}\left(-e^{-t}\right) d t=\int_{-\infty}^{+\infty} e^{-z t} e^{-e^{-t}} d t .
$$

Observe that $\Gamma(-i z)=\int_{-\infty}^{+\infty} e^{i z t} e^{-e^{-t}} d t$ is, according to Theorem 5.5, the positive definite holomorphic function on $S_{0,+\infty}$ associated with the measure $\mu_{0}$ such that $d \mu_{0}(t)=e^{-e^{-t}} d t$. Then, in view of Proposition $4.5, \Gamma(z)$ is co-positive definite on $T_{0,+\infty}$.

For each integer $n \geq 0$ and each vertical strip $T_{-n-1,-n}$ we may rewrite the expression in Example 3 in the form

$$
\Gamma(z)=\int_{0}^{\infty} e^{z \ln x} \frac{\left(\sum_{m=n+1}^{\infty} \frac{(-1)^{m}}{m !} x^{m}\right)}{x} d x
$$

Using once again the change of variables $t=-\ln x$ we obtain, on each vertical strip $T_{-n-1,-n}$,

$$
\Gamma(z)=\int_{-\infty}^{+\infty} e^{-z t}\left(\sum_{m=n+1}^{\infty} \frac{(-1)^{m}}{m !} e^{-m t}\right) d t
$$

Reasoning as in the first case, we conclude that $\Gamma(z)$ is a co-positive or co-negative definite function on each of the vertical strips $T_{-n-1,-n}$ associated, for $n \geq 0$, with measures $\mu_{n+1}$ such that $d \mu_{n+1}(t)=\left(\sum_{m=n+1}^{\infty} \frac{(-1)^{m}}{m !} e^{-m t}\right) d t$. Each of these measures is exponentially finite with respect to the interval $]-n-1,-n[$. Their respective signs are determined by the sign of the factor $(-1)^{n+1}$ corresponding to the first term of the $n$th order tail of the series expansion of $e^{x}$.

Example 10 Consider the normalized Bessel function $\mathcal{J}_{\alpha}$ defined in Example 4. Observe that, for $z \in \mathbb{C}$, its integral representation may be written in the form

$$
\begin{aligned}
\mathcal{J}_{\alpha}(z) & =\int_{-1}^{1} \frac{1}{2^{\alpha} \sqrt{\pi} \Gamma(\alpha+1 / 2)}\left(1-t^{2}\right)^{\alpha-1 / 2} e^{i z t} d t \\
& =\int_{-\infty}^{+\infty} \sigma_{\mathcal{J}_{\alpha}}(t) e^{i z t} d t
\end{aligned}
$$

where

$$
\sigma_{\mathcal{J}_{\alpha}}(t)= \begin{cases}\frac{\left(1-t^{2}\right)^{\alpha-1 / 2}}{2^{\alpha} \sqrt{\pi} \Gamma(\alpha+1 / 2)} & \text { if }-1 \leq t \leq 1 \\ 0 & \text { otherwise. }\end{cases}
$$

Notice that $\sigma_{\mathcal{J}_{\alpha}}$ is the density function of a non-negative measure $\mu_{\mathcal{J}_{\alpha}}$. Since $\sigma_{\mathcal{J}_{\alpha}}$ has compact support, $\mu_{\mathcal{J}_{\alpha}}$ is exponentially finite with respect to the interval ] $-\infty,+\infty[$ 
and the corresponding Fourier-Laplace integral

$$
\mathcal{J}_{\alpha}(z)=\int_{-\infty}^{+\infty} e^{i z t} d \mu_{\mathcal{J}_{\alpha}}(t)
$$

represents, by Theorem 5.5, an entire positive definite function. Since, in the special case $\alpha=0$, we have $J_{0}(z) \equiv \mathcal{J}_{0}(z)$, we derive the same conclusion for the Bessel function $J_{0}(z)$. Notice that, in this case, we have

$$
J_{0}(z)=\mathcal{F} \mathcal{L}\left(\mu_{J_{0}}\right)
$$

where $\mu_{J_{0}}$ admits the density function

$$
\sigma_{J_{0}}(t)= \begin{cases}\frac{1}{\pi \sqrt{1-t^{2}}} & \text { if }-1 \leq t \leq 1 \\ 0 & \text { otherwise }\end{cases}
$$

Example 11 Recall from Example 5 that the Riemann $\zeta$ function is co-positive definite on the half-plane $T_{1,+\infty}$, where it is defined by $\zeta(z)=\sum_{n \geq 1} \frac{1}{n^{z}}$. Hence, according to Theorem 5.5 and Proposition 4.5, the $\zeta$ function is associated with a non-negative measure $\mu$ which is exponentially finite with respect to the interval $] 1,+\infty[$. We now proceed to construct this measure.

Denote by $\varepsilon_{x}$ the unit mass at $x$, by which we mean the Borel measure such that $\varepsilon_{x}(A)=\int_{A} d \varepsilon_{x}=\left\{\begin{array}{ll}0 & \text { if } x \notin A \\ 1 & \text { if } x \in A\end{array}\right.$ for every Borel set $A$. Defining $\mu=\sum_{n=1}^{\infty} \varepsilon_{\log n}$, we obtain

$$
\begin{aligned}
\mathcal{F} \mathcal{L}(\mu)(i z) & =\int_{-\infty}^{+\infty} e^{-z t} d \mu(t) \\
& =\int_{-\infty}^{+\infty} e^{-z t} d\left(\sum_{n=1}^{\infty} \varepsilon_{\log n}\right) \\
& =\sum_{n=1}^{\infty} e^{-z \ln n} \\
& =\zeta(z) .
\end{aligned}
$$

The uniqueness assertion in Theorem 5.5 implies $\mu$ is the only measure associated with $\zeta$ in this way.

Example 12 We now consider an interesting analytic extension of the integral representation of the $\zeta$ function obtained above to the half-plane $\Re(z)>0$. Define, for all non-negative $s$ and $t, \mathbf{1}_{[0, t]}(s)=\left\{\begin{array}{ll}1 & \text { if } 0 \leq s \leq t \\ 0 & \text { otherwise }\end{array}\right.$. Letting $\mu$ be the measure constructed in example 11 , observe that $\int_{0}^{t} d \mu(s)=\mu([0, t])=\left[e^{t}\right]$, where [.] denotes 
the integer part of a real number. We then have, for $\Re(z)>1$ :

$$
\begin{aligned}
z \int_{0}^{+\infty}\left[e^{t}\right] e^{-z t} d t & =\int_{0}^{+\infty} z \mu([0, t]) e^{-z t} d t \\
& =\int_{0}^{+\infty} \int_{0}^{+\infty} \mathbf{1}_{[0, t]}(s) d \mu(s) z e^{-z t} d t \\
& =\int_{0}^{+\infty} \int_{0}^{+\infty} \mathbf{1}_{[0, t]}(s) z e^{-z t} d t d \mu(s) \\
& =\int_{0}^{+\infty} \int_{s}^{+\infty} z e^{-z t} d t d \mu(s) \\
& =\int_{0}^{+\infty}-\left.e^{-z t}\right|_{s} ^{+\infty} d \mu(s) \\
& =\int_{0}^{+\infty} e^{-z s} d \mu(s) \\
& =\zeta(z) .
\end{aligned}
$$

where we have used Fubini in the third equality. We denote as usual by $\{x\}=x-[x]$ the fractional part of a real number $x$. Observing that $\int_{0}^{+\infty} e^{(1-z) t} d t=\frac{1}{z-1}$ for $\Re(z)>1$, we derive from (5.3) that

$$
\zeta(z)=\frac{z}{z-1}-z \int_{0}^{+\infty}\left\{e^{t}\right\} e^{-z t} d t .
$$

We then have

$$
\zeta(z)=\frac{z}{z-1}-z \mathcal{U}(z)
$$

where $\mathcal{U}(z)=\int_{0}^{+\infty}\left\{e^{t}\right\} e^{-z t} d t$. Since $\left\{e^{t}\right\}$ is bounded, we conclude that

$$
\sigma_{\mathcal{U}}(t)= \begin{cases}\left\{e^{t}\right\} & \text { if } t \geq 0 \\ 0 & \text { otherwise }\end{cases}
$$

is the density function associated with a non-negative measure $\mu_{\mathcal{U}}$ which is exponentially finite with respect to the interval ]0, $+\infty\left[\right.$. Hence $\mathcal{U}(z) \equiv \mathcal{F} \mathcal{L}\left(\mu_{\mathcal{U}}\right)(i z)$ is a holomorphic co-positive definite function on the half-plane $\Re(z)>0$ and, by definition of $\zeta(z)$, the meromorphic right hand side of (5.4) coincides, on this set, with $\zeta(z)$.

Example 13 Recall the function $\mathcal{Z}=-\zeta(z) / z$ defined by (4.2) in Example 6. According to (5.3) we have

$$
\mathcal{Z}(z)=\int_{-\infty}^{+\infty}-\left[e^{t}\right] e^{-z t} d t \text { for } \Re(z)>1
$$


On the other hand, we derive from (5.4) that

$$
\mathcal{Z}(z)=\frac{1}{1-z}+\mathcal{U}(z)=\frac{1}{1-z}+\int_{0}^{+\infty}\left\{e^{t}\right\} e^{-z t} d t
$$

for $\Re(z)>0$ and $z \neq 1$. Observing that

$$
\frac{1}{1-z}=\int_{-\infty}^{0} e^{t} e^{-z t} d t \text { for } \Re(z)<1
$$

we may write

$$
\mathcal{Z}(z)=\int_{-\infty}^{+\infty}\left\{e^{t}\right\} e^{-z t} d t \text { for } 0<\Re(z)<1
$$

Hence we conclude that $\mathcal{Z}(z)$ is a holomorphic co-negative definite function on the half-plane $\Re(z)>1$ and a holomorphic co-positive definite function on the vertical strip $0<\Re(z)<1$. Accordingly, the non-negative measure $-\mu_{\mathcal{Z}}^{r}\left(\right.$ resp. $\left.\mu_{\mathcal{Z}}^{l}\right)$ associated with the density function $-\sigma_{\mathcal{Z}}^{r}=\left[e^{t}\right]$ (resp. $\sigma_{\mathcal{Z}}^{l}=\left\{e^{t}\right\}$ ) is exponentially finite with respect to the interval $] 1,+\infty[$ ) (resp. $] 0,1[$ ).

Notice the interesting fact that the zeros of $\zeta(z)$ and $\mathcal{Z}(z)$ coincide. Hence we conclude, using the arguments in Example 5, that $\mathcal{Z}$ is neither co-positive nor conegative definite in any cosum set contained in the half-plane $\Re(z)<0$.

Remark 5.6 We remark that Example 13 proves the following integral representation for the $\zeta$ function on the critical strip:

$$
\zeta(z)=-z \int_{-\infty}^{+\infty}\left\{e^{t}\right\} e^{-z t} d t, \quad 0<\Re(z)<1
$$

A change of variable leads to the equivalent integral representation

$$
\zeta(z)=-z \int_{0}^{+\infty} t^{z-1}\{1 / t\} d t, \quad 0<\Re(z)<1,
$$

which is well-known in the literature (Titchmarsh [33], pg. 14, eq. 2.1.5).

This collection of examples shows that meromorphic (co-)positive definite functions defined on maximal horizontal (vertical) strips of holomorphy may have a rather subtle behavior with respect to definiteness upon crossing of this strip. Indeed, they may enter a new maximal holomorphy strip of (co-) positive definiteness; they may enter a maximal holomorphy strip of (co-) negative definiteness, as happens with the $\Gamma$ function; or they may simply enter a region of the complex plane where definiteness properties simply disappear, as happens with the $\zeta$ function in the half-plane $\Re(z)<1$. An interesting problem, open at the time of writing, is to characterize these transitions. 


\section{References}

1. Askey, R.: Grünbaum's inequality for Bessel functions. J. Math. Anal. Appl. 293, 122-124 (1973)

2. Baricz, A.: Generalized Bessel Functions of the First Kind, vol. 1994. Springer, Berlin (2010)

3. Berg, C., Christensen, J., Ressel, P.: Harmonic Analysis on Semigroups, vol. 100. Springer, New York (1984)

4. Bernstein, S.: Sur les fonctions absolument monotones. Acta Math. 52, 1-66 (1929)

5. Bisgaard, T., Sasvàri, Z.: Characteristic Functions and Moment Problems. Nova Science Publishing, New York (2000)

6. Buescu, J., Paixão, A.: Positive definite matrices and integral equations on unbounded domains. Differ. Integr. Equ. 19(2), 189-210 (2006)

7. Buescu, J., Paixão, A., Garcia, F., Lourtie, I.: Positive-definiteness, integral equations and Fourier transforms. J. Integr. Equ. Appl. 16(1), 33-52 (2004)

8. Buescu, J., Paixão, A.: A linear algebraic approach to holomorphic reproducing kernels in $\mathbb{C}^{n}$. Linear Algebra Appl. 412(2-3), 270-290 (2006)

9. Buescu, J., Paixão, A.: Positive definite matrices and differentiable reproducing kernel inequalities. J. Math. Anal. Appl. 320, 279-292 (2006)

10. Buescu, J., Paixão, A.: On differentiability and analyticity of positive definite functions. J. Math. Anal. Appl. 375(1), 336-341 (2011)

11. Buescu, J., Paixão, A.: Real and complex variable positive definite functions. São Paulo J. Math. Sci. 6(2), 155-169 (2012)

12. Buescu, J., Paixão, A.: Complex variable positive definite functions. Complex Anal. Oper. Theory 8(4), 937-954 (2014)

13. Buescu, J., Paixão, A., Symeonides, A.: Complex positive definite functions on strips. Complex Anal. Oper. Theory 11(3), 627-649 (2017)

14. Buescu, J., Paixão, A., Oliveira, C.: Propagation of regularity and positive definiteness: a constructive approach. Z. Anal. Anwend. 37(1), 1-24 (2018)

15. Devinatz, A.: Integral representations of positive definite functions. Trans. Am. Math. Soc. 74, 56-77 (1953)

16. Devinatz, A.: Integral representations of positive definite functions II. Trans. Am. Math. Soc. 77, 455-480 (1954)

17. Donoghue, W.: Distributions and Fourier Transforms. Academic Press, New York (1969)

18. Ehm, W., Genton, M., Gneiting, T.: Stationary covariances associated with exponentially convex functions. Bernoulli 9(4), 607-615 (2003)

19. El Kamel, J., Mehrez, K.: A function class of strictly positive definite and logarithmically completely monotonic functions related to the modified Bessel functions. Positivity 22(5), 1403-1417 (2018)

20. Graczyk, P., Loeb, J.: Bochner and Schoenberg theorems on symmetric spaces in the complex case. Bull. Soc. Math. France 122(4), 571-590 (1994)

21. Jorgensen, P., Niedzialomski, R.: Extension of positive definite functions. J. Math. Anal. Appl. 422(1), $712-740(2015)$

22. Jorgensen, P., Pedersen, S., Tian, F.: Feng Extensions of Positive Definite Functions. Applications and Their Harmonic Analysis. Lecture Notes in Mathematics, vol. 2160. Springer, New York (2016)

23. Kosaki, H.: Positive definiteness of functions with applications to operator norm inequalities. Mem. Am. Math. Soc. 212, 997 (2011)

24. Lax, P.: Functional Analysis. Wiley, New York (2002)

25. Loeb, J., Youssfi, E.: Fonctions holomorphes définies positives sur les domaines tubes. C. R. Math. Acad. Sci. Paris 343(2), 87-90 (2006)

26. Lukacs, E.: Characteristic Functions, 2nd edn. Griffin and Co., Helsinki (1970)

27. Neuman, E.: Inequalities involving Bessel functions of the first kind. J. Inequal. Pure Appl. Mat 5(4), article 94, 4pgs (electronic)

28. Rudin, W.: Fourier Analysis on Groups. Interscience Publishers, Wiley (1962)

29. Stewart, J.: Positive definite functions and generalizations, an historical survey. Rocky Mt. J. Math. 6(3), 409-434 (1976)

30. Sasvàri, Z.: Positive Definite and Definitizable Functions. Mathematical Topics, vol. 2. Akademie Verlag, Berlin (1994)

31. Sasvàri, Z.: Multivariate Characteristic and Correlation Functions. De Gruyter Studies in Mathematics, vol. 50. Walter de Gruyter \& Co., Berlin (2013) 
32. Selinger, V.: Geometric properties of normalized Bessel functions. Pure Math. Appl. 6, 273-277 (1995)

33. Titchmarsh, E.C.: The Theory of the Riemann Zeta-Function, 2nd edn. Oxford University Press, Oxford (1986)

34. Watson, G.: A Treatise on the Theory of Bessel Functions. Cambridge U. P, Cambridge (1944)

35. Whittaker, E., Watson, G.: A Course in Modern Analysis, 4th edn. Cambridge U. P, Cambridge (1996)

36. Widder, D.: Necessary and sufficient conditions for the representation of a function by a doubly infinite Laplace integral. Bull. Am. Math. Soc. 40(4), 321-326 (1934)

37. Youssfi, E.: Harmonic analysis on conelike bodies and holomorphic functions on tube domains. J. Funct. Anal. 155(2), 381-435 (1998)

38. Zastavnyi, V.: On positive definiteness of some functions. J. Multivar. Anal. 73, 55-81 (2000)

Publisher's Note Springer Nature remains neutral with regard to jurisdictional claims in published maps and institutional affiliations. 